\title{
Dynamic response of a cracked rotor-bearing-seal system
}

\author{
Huang Zhiwei* \\ National Key Laboratory on Ship Vibration \& Noise, China Ship Development and Design Centre, Wuhan 430064 China
}

\begin{abstract}
With the structural parameters of the rotation machinery increased, the seal fluid induced force imposed on the rotor will significantly increase. Taking a cracked rotor-bearing-seal system as object the non-linear dynamic behaviours of the coupled system are investigated using numerical integration method. For the crack in shaft, the segmented switch function is applied to express the process of the crack's opening and closing in rotation, and the cross stiffness originated from crack is considered. Various nonlinear phenomena compressing periodic, quasi-periodic and chaotic motions in the rotor system are analysed. The research results show that the unstable form of the rotor system is Hopf bifurcation when the crack depth is smaller. The influence to the response of the system increased along with the crack depth, the unstable form of the coupled system is period-doubling bifurcation. The seal clearance has an important impact on stability of the cracked rotor system. In other words, the fluid induced force can reduce the periodic responses of the rotor. It is indicated that this study can contribution to a further understanding of the nonlinear of such a rotor-bearing system with crack and seal.
\end{abstract}

\section{Introduction}

Recently the demand for more powerful and efficient rotating machinery has led to higher operating speed and working pressure, which results in the need of reliable components such as bearings and seals. Not only in bearing, but also in seals, the shaft motion can induce rotor dynamic forces that may lead to self-excited vibration of the shaft [1]. With the increase in the rotating speed, the performances of the rotor system are significantly influenced by the characteristics of the seal [2]. Under these circumstances, the rotor is likely to develop one or more faults, e.g. crack, bow, etc. However, Fatigue cracks, because of their potential to cause catastrophic failures, are a grave threat to an uninterrupted operation and performance of the modern machines [3-4]. So it is imperative to understand the nonlinear behaviour of the rotor-seal and rotor-crack interaction in the design of the system. And recently, the coupling effect of multi-nonlinear factors on rotor system is a new focus.

Literatures related to rotor-bearing-seal system with crack phenomena are abundant. Many theoretical studies numerical calculations and measurements have been carried out to determine the influence of the dynamic vibration due to seal or crack. A recent method to identify both the excitation and flexible support parameters of a rotor-bearing-foundation system have been verified experimentally [5]. Ding [6] and Jing [7] investigated the Hopf bifurcation behaviour and dynamic characteristics of a symmetric rotor-seal system. Li [8] studied the dynamic stability of rotor system with labyrinth seal based on the adopted model and Newmark integration method. Nonlinear dynamic and stability of

\footnotetext{
* Corresponding author: hzwhust@aliyun.com
}

the rotor-bearing-seal system were investigated both theoretically and experimentally by Shen [9]. The CFD method and a rotating frame were applied to analyse the influence of different steam parameters on the leakage characteristics and dynamic characteristic coefficients [10]. Muszynska's nonlinear seal fluid dynamic force model was used in these rotor systems above. As for the seal model, the Muszynska model [11] not only reflects the nonlinear factors in seals but also has a clear physical meaning. And it is employed in this study.

Under fatigue load operating conditions, the stiffness and dynamic behaviour of the rotor shaft will change greatly and these crack faults may cause huge failure and loss. Zeng [12] and Sekhar [13] calculated stiffness of a simple rotor with a transverse crack and investigated the relationships between the stiffness and some parameters including the crack location, the crack depth and the slenderness ratio, etc. Several researchers studied imbalance vibration response of the cracked rotors and found that along with increase in $1 \mathrm{X}$ frequency component, rotor crack induced periodic steady-state lateral vibrations at $2 \mathrm{X}$ and $3 \mathrm{X}$ frequency components due to breathing of the crack under gravity loading. The vibration in crack direction determines the breathing of the crack. In switching crack model, the crack can be considered to either fully open or fully closed when the crack depth is very small. However, partial opening and closing of the crack is present as the crack depth increasing [12]. Thus, the cross-coupled stiffness terms can't be negligible.

However, in almost all published papers, only a single factor, seal fluid force or transverse crack, was taken into account in studying the dynamics of a rotor system. The method will cause the results to deviate 
from the facts. Therefore, it is necessary to consider the rotor, bearings, seals and crack as a whole system and model them in one numerical model. So, an in-depth study investigating the vibration characteristic of a cracked rotor-bearing-seal system is the main objective of this paper.

\section{Mathematical model}

The key point in the paper is to analyze the influence of the crack and seal. Firstly, an assumption is made that the torsional vibration of rotor may be neglected and only the transverse vibration of rotor is considered. Therefore, the rotor-bearing system can be modeled as a Jeffcott rotor system, in which the rotor is simplified to one disk and the masses of the shaft equivalent to the disk and two bearings. The model of rotor-bearing system is shown in Fig. 1, where $O_{1}$ is the bearing center, $\mathrm{O}_{2}$ is the disk geometric center, $\mathrm{O}_{3}$ is the disk mass center, $F_{x}$ and $F_{y}$ are seal fluid forces, $f_{x}$ and $f_{y}$ are dimensionless oil forces, $m_{1}$ is the bearing mass, $m_{2}$ is the disk mass, $c$ is the clearance of bearing, $R$ is the radius of shaft, $a$ is the crack depth in middle of shaft, $k$ is the stiffness of the shaft without crack and $\delta$ is an initial clearance between the rotor and stator.

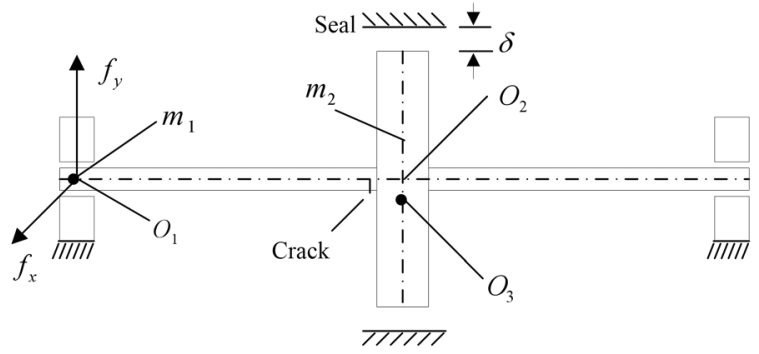

Fig.1 A cracked rotor-bearing-seal system

\subsection{Governing equations}

In the mathematical model of the rotor-bearing system, four degrees of freedom-horizontal and vertical displacements of the rotor are taken into consideration at the disk location $\left(x_{2}, y_{2}\right)$ and at the journal $\left(x_{1}, y_{1}\right)$, correspondingly. If $X_{1}=x_{1} / c, Y_{1}=y_{1} / c, X_{2}=x_{2} / c$ and $Y_{2}=y_{2} / c$, then the dynamic equations of system can be established as follows:

$$
\begin{aligned}
& \left\{\begin{array}{l}
\ddot{X}_{1} \\
\ddot{Y}_{1}
\end{array}\right\}+\frac{c_{1}}{\omega m_{1}}\left\{\begin{array}{l}
\dot{X}_{1} \\
\dot{Y}_{1}
\end{array}\right\}+\frac{k}{\omega^{2} m_{1}}\left[\begin{array}{ll}
k_{x x} & k_{x y} \\
k_{y x} & k_{y y}
\end{array}\right]\left\{\begin{array}{l}
X_{1}-X_{2} \\
Y_{1}-Y_{2}
\end{array}\right\} \\
& =\frac{\sigma}{c \omega^{2} m_{1}}\left[\begin{array}{c}
f_{x}\left(X_{1}, Y_{1}\right) \\
f_{y}\left(X_{1}, Y_{1}\right)
\end{array}\right]+\frac{1}{c \omega^{2}}\left[\begin{array}{l}
0 \\
g
\end{array}\right]
\end{aligned}
$$

$$
\begin{aligned}
& \left\{\begin{array}{l}
\ddot{X}_{2} \\
\ddot{Y}_{2}
\end{array}\right\}+\frac{c_{2}}{\omega m_{2}}\left\{\begin{array}{l}
\dot{X}_{2} \\
\dot{Y}_{2}
\end{array}\right\}+\frac{2 k}{\omega^{2} m_{2}}\left[\begin{array}{ll}
k_{x x} & k_{x y} \\
k_{y x} & k_{y y}
\end{array}\right]\left\{\begin{array}{l}
X_{2}-X_{1} \\
Y_{2}-Y
\end{array}\right\} \\
& =\frac{e}{c}\left[\begin{array}{c}
\cos \omega t \\
\sin \omega t
\end{array}\right]+\frac{1}{c \omega^{2} m_{2}}\left[\begin{array}{l}
F_{x}\left(X_{2}, Y_{2}\right) \\
F_{y}\left(X_{2}, Y_{2}\right)
\end{array}\right]+\frac{1}{c \omega^{2}}\left[\begin{array}{l}
0 \\
g
\end{array}\right]
\end{aligned}
$$

Where $c_{1}$ is damping coefficient of the bearing, $c_{2}$ is the viscous damping of the rotor disk, $e$ is the eccentricity of the rotor, $\omega$ is the angular speed of the rotor, $\sigma$ is Summerfield modifying parameter and $\sigma=\mu \omega R L\left(\frac{R}{c}\right)^{2}\left(\frac{L}{2 R}\right)^{2}$.

Considering the nonlinear oil-film force model under the assumption of short bearing, a dynamic model of the nonlinear oil-film force is established for better accuracy and convergence. The non-dimension oil-film force $f_{x}$ and $f_{y}$ are obtained from bearing theory and it can be expressed as ${ }^{[9]}$.

\section{2 crack model}

The periodic closing and opening of the crack is called "breathing" action. Its breathing motion can be described by the steering function $f(\Psi)$ related to the crack depth ${ }^{[12]}$. For small cracks when $A<0.5$ (dimensionless $A=a / R$ ), Gash [14] considered the simple hinge model as a good model. However, as the increase of crack depth, when $A \geq 0.5$, the model can accurately reflect the change of breathing crack by Mayes. Then it is expressed as

$$
f(\psi)= \begin{cases}\frac{1}{2}+\frac{2}{\pi} \cos \psi-\frac{2}{3 \pi} \cos 3 \psi+\frac{2}{5 \pi} \cos 5 \psi & A<0.5 \\ \frac{1}{2}(1+\cos \psi) & \mathrm{A} \geq 0.5\end{cases}
$$

Where the rotating angle satisfies $\psi=\omega t+\phi_{0}+\beta-\phi, \beta$ is the phase between the eccentricity and the center of the crack, i.e. crack angle and $\phi$ is expressed as

$\phi=\left\{\begin{array}{l}\operatorname{arctg}(y / x)+2 n \pi \\ \operatorname{arctg}(y / x)+(2 n+1) \pi \\ \pi / 2+2 n \pi \\ 3 \pi / 2+2 n \pi\end{array} \quad \mathrm{n}=0, \pm 1, \pm 2, \ldots\right.$

Since an exact model of the "breathing" crack is quite complicated, the variation of stiffness of the crack shaft in the rotating co-ordinate system is often considered in the form

$$
\begin{aligned}
& {\left[\begin{array}{ll}
k_{x x} & k_{x y} \\
k_{y x} & k_{y y}
\end{array}\right]=\left[\begin{array}{ll}
1 & 0 \\
0 & 1
\end{array}\right]-f(\psi) \times} \\
& {\left[\begin{array}{ll}
k_{\zeta} \cos ^{2} \omega t+k_{\eta} \sin ^{2} \omega t & \left(k_{\zeta}-k_{\eta}\right) \sin \omega t \cos \omega t \\
\left(k_{\zeta}-k_{\eta}\right) \sin \omega t \cos \omega t & k_{\zeta} \sin ^{2} \omega t+k_{\eta} \cos ^{2} \omega t
\end{array}\right]}
\end{aligned}
$$

Where $k_{\zeta}$ is the stiffness in $\zeta$ orientation in rotating coordinates, $k_{\eta}$ is the stiffness in $\eta$ orientation in rotating co-ordinates[6]. 


\subsection{Nonlinear seal fluid forces}

According to Muszynska's force model, the nonlinear character of seal fluid force may be expressed as follows:

$$
\begin{aligned}
& \left\{\begin{array}{c}
F_{x} \\
F_{y}
\end{array}\right\}=-\left[\begin{array}{cc}
K-m_{f} \tau^{2} \omega^{2} & \tau \omega D \\
-\tau \omega D & K-m_{f} \tau^{2} \omega^{2}
\end{array}\right]\left\{\begin{array}{l}
x\} \\
y
\end{array}\right\}- \\
& {\left[\begin{array}{cc}
D & 2 \tau m_{f} \omega \\
-2 \tau m_{f} \omega & D
\end{array}\right]\left\{\begin{array}{l}
\dot{x} \\
\dot{y}
\end{array}\right\}-\left[\begin{array}{cc}
m_{f} & 0 \\
0 & m_{f}
\end{array}\right]\left\{\begin{array}{l}
\ddot{x} \\
\ddot{y}
\end{array}\right\}}
\end{aligned}
$$

Muszynska model assumes that the fluid force is rotating with angular velocity $\tau \omega$, where $\tau$ is the fluid average circumferential velocity ratio ${ }^{[11]}$. In Equation (6), $K, D$ and $m_{f}$ are fluid stiffness, damping and inertia coefficients respectively. The vary $K, D$ and $\tau$ vary with increasing of journal eccentricity and can be expressed as follows

$$
\left\{\begin{array}{l}
K=K_{0}\left(1-e^{2}\right)^{-n} \\
D=D_{0}\left(1-e^{2}\right)^{-n} \quad n=0.5 \sim 3,0<b<1 \\
\tau=\tau_{0}(1-e)^{b}
\end{array}\right.
$$

Where the relative eccentricity $e=\sqrt{x^{2}+y^{2}} / \delta$, and $n$, $b, \tau_{0}$ are determined by material seal. Generally $\tau_{0}$ is less than 0.5. The Muszynska model of seal fluid forces is applied to investigate the stability of rotor. The coefficients $K_{0}, D_{0}$ and $\tau_{0}$, which are involved in seal length, seal clearance, pressure margin and seal radius, are calculated with Black-Childs Equation ${ }^{[2]}$.

\section{Simulation results and analysis}

The rotating speed is one of the primary parameters affecting the dynamic characteristics of a rotor system. Dynamic responses of the cracked rotor-bearing-seal system become more complicated with rotating speed as a control parameter when the nonlinear oil film force is combined with the nonlinear seal fluid force. The main partial parameters of numerical calculation are as follows:

$m_{1}=4.0 \mathrm{~kg}, m_{2}=32.1 \mathrm{~kg}, \quad c_{1}=1050 \mathrm{~N} \cdot \mathrm{s} / \mathrm{m}, \quad c_{2}=2100 \mathrm{~N} \cdot \mathrm{s} / \mathrm{m}$, $e=0.05 \mathrm{~mm}, \quad c=0.11 \mathrm{~mm}, \quad R=25 \mathrm{~mm}, \quad L=12 \mathrm{~mm}$, $\mu=0.018 \mathrm{~Pa} \cdot \mathrm{s}, \quad k=2.5 \times 10^{7} \mathrm{~N} / \mathrm{m}, \quad \beta=\pi / 2, b=0.2, n=2.5$, $n_{0}=0.079, m_{0}=-0.25, \tau_{0}=0.5, \xi=0.1, R_{s}=0.16 \mathrm{~m}, l=0.06 \mathrm{~m}$, $v=5.0 \mathrm{~m} / \mathrm{s}, \gamma=1.3 \times 10^{-3} \mathrm{~Pa} \cdot \mathrm{s}, \Delta P=2.0 \times 10^{5} \mathrm{~Pa}$.

\subsection{Effect of crack depth}

Fig. 2(a) (c) gives the bifurcation diagrams of the rotor system in the rotating speed range $\omega \in[100,2100] \mathrm{rad} / \mathrm{s}$ at different crack depth, where $\delta=1 \mathrm{~mm}$. It can be observed that the influence of crack depth to the vibration of the rotor system is obvious. As shown in Fig. 2(a), the system responses display synchronous motion with period-one, double-periodic motion, quasi-periodic motion and chaotic motion. Fig. 3(a) shows the dynamic characteristics of the rotor at $\omega=1400 \mathrm{rad} / \mathrm{s}$. The Poincaré map indicates that the motion is chaotic on account of the fractal like structure of the attractor, and the spectrum for the chaotic motion is not composed of discrete frequencies but displays a continuous, broad band, nature at the lower frequencies. As rotating speed $\omega$ increasing, transitory quasi-periodic motion occurs from period-two to period-five. At $\mathrm{A}=0.6$, the bifurcation diagram of the rotor system is very similar to the former one. Fig. 3(b) illustrates the dynamic characteristics in the chaotic region at $\omega=1020 \mathrm{rad} / \mathrm{s}$. There are four islands in the Poincaré map and only some low-frequencies with large amplitude. It can be observed from Fig. 2(a) and (b) that for the rotor a sequence of Hopf bifurcations leads the system enter into chaotic regime, and then it experiences a sequence of reverse Hopf bifurcations. The influence to the response of the system increased along with the crack depth, and the unstable form of the coupled system is period-doubling bifurcation. When A increases to 0.8 , the region of chaotic motion is divided to two islands caused by the crack and the attractors become self-similar structure in the Poincaré map, as shown in Fig. 3(c). Although the influence of the oil film force is greater than that of the crack in supercritical speed range, there are some other synchronous motions such as period-eight. With the increase of rotation speed, there is a long region for quasi-periodic within supercritical speed range due to the nonlinear oil film forces.

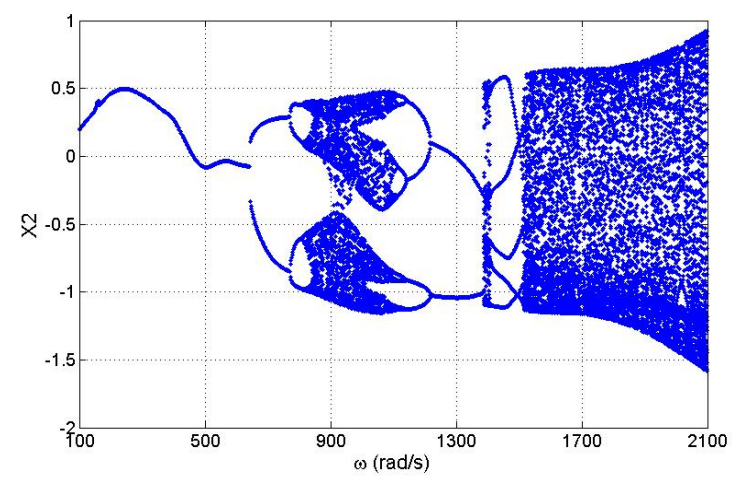

(a) $\mathrm{A}=0.4$

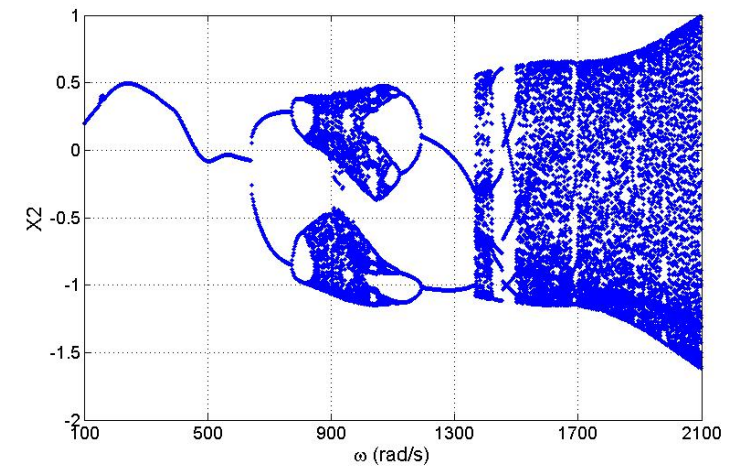

(b) $\mathrm{A}=0.6$ 


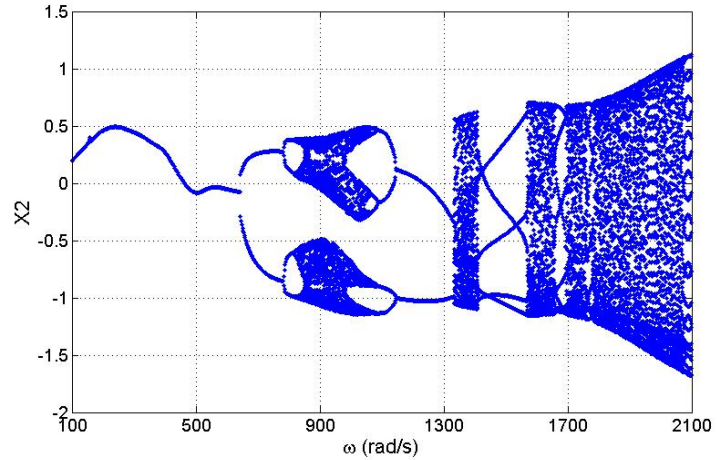

(c) $\mathrm{A}=0.8$

Fig.2 Bifurcation diagrams of the rotor response with $\omega$ as a control parameter at different dimensionless crack depth
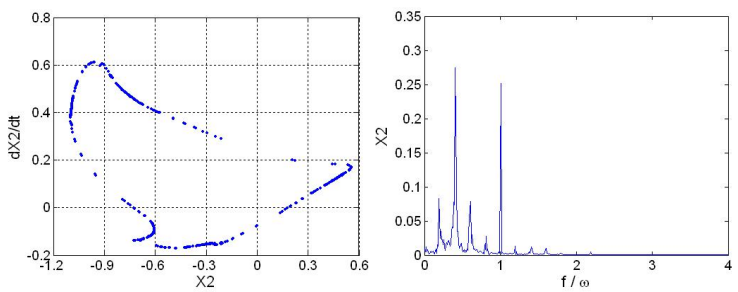

(a) $A=0.4$ and $\omega=1400 \mathrm{rad} / \mathrm{s}$
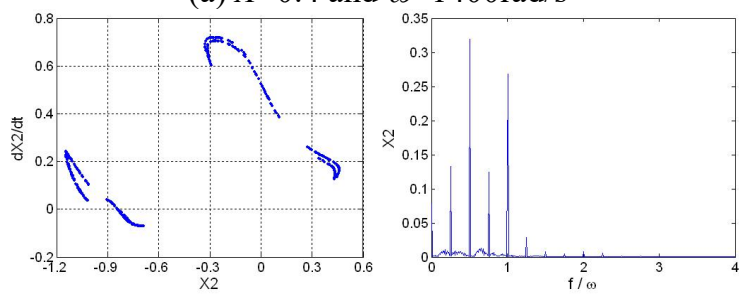

(b) $A=0.6$ and $\omega=1020 \mathrm{rad} / \mathrm{s}$
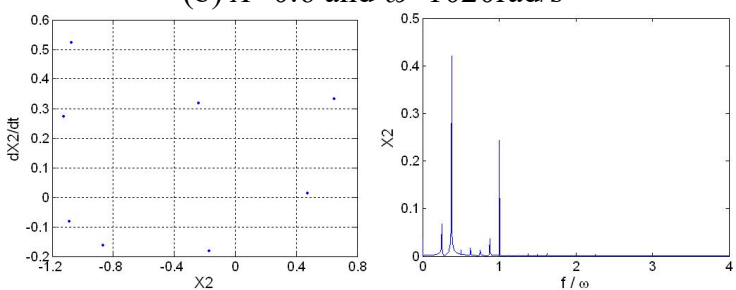

(c) $A=0.8$ and $\omega=1680 \mathrm{rad} / \mathrm{s}$

Fig 3 Poincaré maps and amplitude spectrum diagrams of the rotor response at different crack depth and rotating speed

Comparing with Fig. 2(a)-(c), it reveals that the critical speed from period-two motion stepping into quasi-periodic motion arrives in advance with the increase of crack depth. In other words, the effect of oil film force is more and more strong.

\subsection{Effect of seal clearance}

It is essential to maximize the seal clearance between the rotor and stator for the stable operation. The seal clearance plays a more important role in the vibration of the rotor system. Fig. 4 gives the bifurcation diagrams of $\omega$ on the response of the rotor system at different seal clearance, where $A=0.7$. It can be seen that the influence of seal clearance is not obvious, but there are a few changes in some speed regions. Two specific cases are now presented to do some in-depth study investigating the behaviour of the model derived above. One is the region for chaotic motion around critical speed, and the other is the region for quasi-periodic motion between period-two and period-five. Fig. 5 shows the Poincaré maps and amplitude spectrum diagrams at $\omega=950 \mathrm{rad} / \mathrm{s}$ and $\omega=1400 \mathrm{rad} / \mathrm{s}$, respectively. It can be observed from the Poincaré maps that the number of the attractors for chaotic and quasi-periodic motions reduces and the structure of the attractors becomes compact as the seal clearance increasing. The spectrums are in a continuous nature at the lower frequencies and some amplitude is larger than that of $1 \mathrm{X}$ component. With the increase of seal clearance, the amplitudes become small at lowfrequencies. Although the seal clearance can make the rotor system away form unstable, it makes adverse effect on vibration of the rotor system.

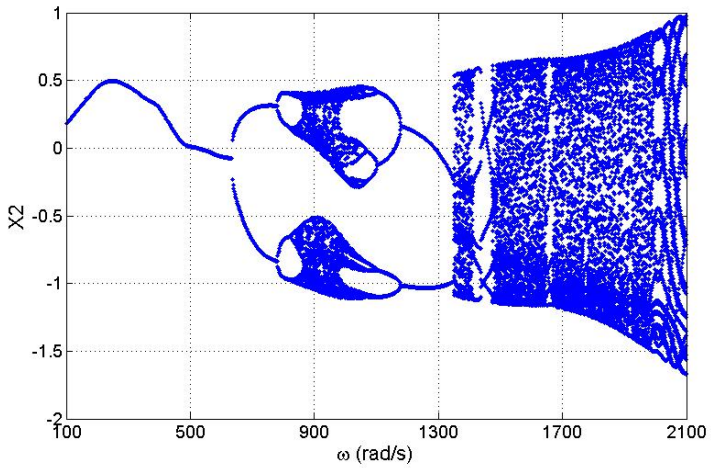

(a) $\delta=2 \mathrm{~mm}$

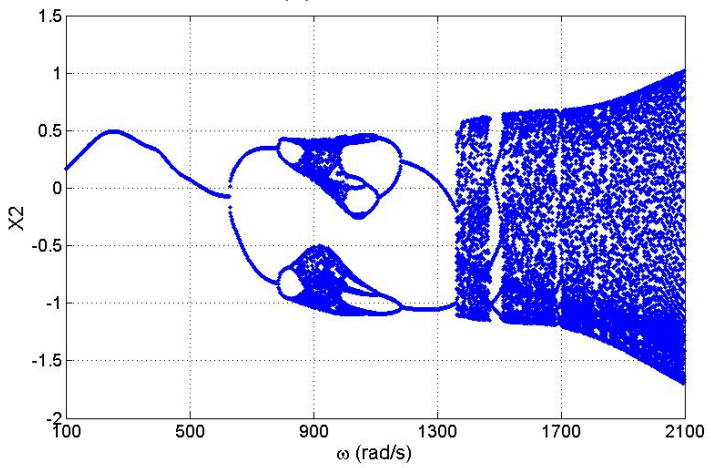

(b) $\delta=3 \mathrm{~mm}$

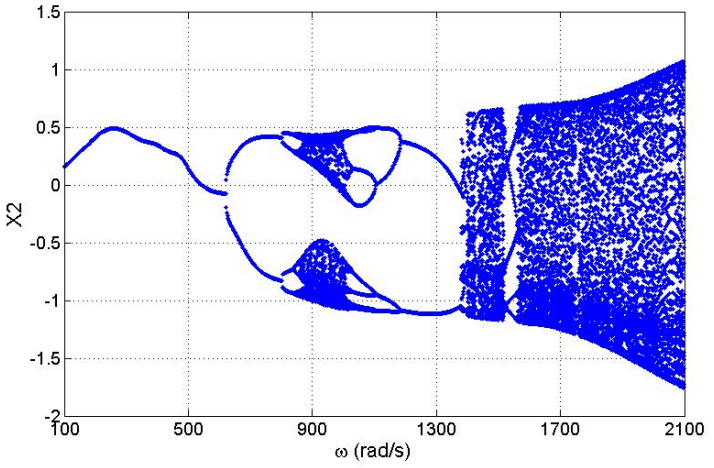

(c) $\delta=5 \mathrm{~mm}$

Fig 4 Bifurcation diagrams of the rotor response with $\omega$ as a control parameter at different seal clearance 

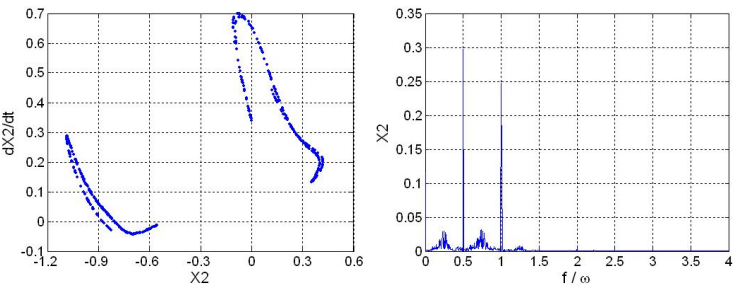

(a) $\delta=2 \mathrm{~mm}$ and $\omega=950 \mathrm{rad} / \mathrm{s}$
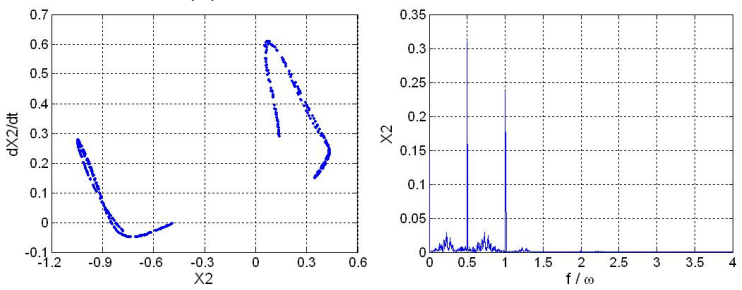

(b) $\delta=5 \mathrm{~mm}$ and $\omega=950 \mathrm{rad} / \mathrm{s}$
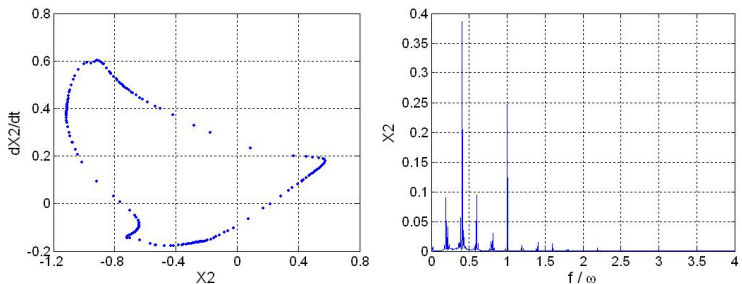

(c) $\delta=2 \mathrm{~mm}$ and $\omega=1400 \mathrm{rad} / \mathrm{s}$
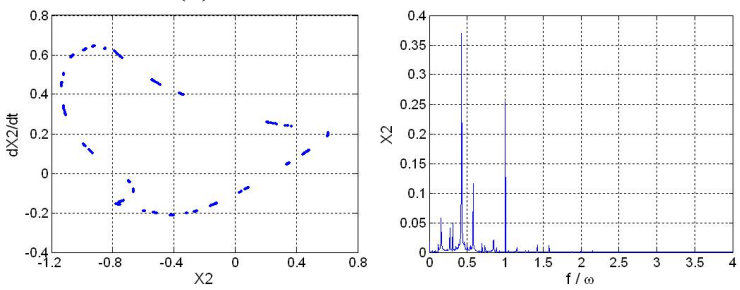

(d) $\delta=5 \mathrm{~mm}$ and $\omega=1400 \mathrm{rad} / \mathrm{s}$

Fig 5 Poincaré maps and amplitude spectrum diagrams of the rotor response at different clearance and rotating speed

\section{Conclusions}

In this paper, a model considering nonlinear seal fluid induced force and transverse crack is put forward to analyse the complicated vibration characteristics of a cracked rotor-bearing-seal system. Special attention is paid to the influence of the crack depth and seal clearance on the bifurcation of the system with rotation speed as a control parameter. Numerical analysis methods were employed to explore dynamic behaviours of the discussed system, including Poincaré maps and bifurcation diagrams. The system exhibits rich forms of periodic, quasi-periodic and chaotic motions. The research results suggest that the unstable form of the rotor system is Hopf bifurcation due to the nonlinear seal force when the depth of crack is smaller. However, with the increase of crack depth, the unstable form of the coupled system becomes period-doubling bifurcation. The seal clearance has an important impact on the stability of cracked rotor system. The spectrum for the chaotic motion is in a continuous nature at the lower frequencies and some amplitude is larger than that of $1 \mathrm{X}$ component. The purpose of the further research is to diagnose the coupling faults, reduce the failure and optimize the design of the rotor-bearing system.

\section{Acknowledgment}

The authors are grateful for supports from the National Natural Science Foundation of China (No.51609226 and No.51879209).

\section{References}

1. M. Cheng, G. Meng, J.P. Jing. Numerical and experimental study of a rotor-bearing-seal system. Mechanism and Machine Theory, 42, 1043-1057 (2007)

2. J. Hua, S. Swaddiwudhipong, Z.S. Liu. Numerical analysis of nonlinear rotor-seal system. Journal of Sound and Vibration, 283, 525-542 (2005)

3. A.K. Darpe. Dynamics of a Jeffcott rotor with slant crack. Journal of Sound and Vibration. 303, 1-28 (2007)

4. F. Fang. Research progress of fault diagnosis methods for rotor bar of induction motor. Chinese Journal of Ship Research, 5(5), 77-81(2010)

5. S. Edwards, A.W. Lees, M.I. Friswell. Experimental identification of excitation and support parameters of a flexible rotor-bearings-Foundation system from a single run-down. Journal of Sound and Vibration. 232(5), 963-992 (2000)

6. Q. Ding, J.E. Cooper, A.Y.T. Leung. Hopf bifurcation analysis of a rotor/seal system. Journal of Sound and Vibration, 252(5), 817-833 (2002)

7. J.P. Jing, G. Meng, Y. Sun. On the non-linear dynamic behavior of a rotor-bearing system. Journal of Sound and Vibration, 274, 1031-1044 (2004)

8. S.T. Li, Q.Y. Xu, X.L. Zhang. Nonlinear dynamic behaviors of a rotor-labyrinth seal system. Nonlinear Dynamic, 47, 321-329 (2007)

9. Z.G. Li, D. Kong, Y.H. Jiao. Research on nonlinear dynamic characteristics of a rotor-seal system. Journal of Vibration and Shock, 28(6), 159-163 (2009)

10. W.Q. HE, Z.H. ZHEGN, W.F. ZHANG. Influence of system parameters on static and dynamic characteristics of labyrinth seal. Chinese Journal of Ship Research, 12(5), 126-131(2017)

11. A. Muszynska, D.E. Bently. Frequency-swept rotating input perturbation techniques and identification of the fluid force models in rotor/bearing/seal systems and fluid handling machines. Journal of Sound and Vibration, 143(1), 103-124 (1990)

12. F. Zeng, Z.T. Wu. Calculating stiffness of a simple rotor with a transverse. Mechanical Science and Technology, 18(5), 745-747 (1999)

13. A.S. Sekhar, J.K. Dey. Effects of cracks on rotor system instability. Mechanism and Machine Theory. $35,1657-1674$ (2000) 
14. R. Gash. A survey of the dynamic behavior of a simple rotating shaft with a transverse crack. Journal of Sound and Vibration, 162, 313-332 (1993) 